\title{
Relationship Between Coronary Plaque Vulnerability and Serum n-3/n-6 Polyunsaturated Fatty Acid Ratio
}

\author{
Toshikazu Kashiyama, MD; Yasunori Ueda, MD, PhD; Takayoshi Nemoto, MD; \\ Mitsuru Wada, MD; Yuki Masumura, MD; Koshi Matsuo, MD; \\ Mayu Nishio, MD, PhD; Akio Hirata, MD, PhD; Mitsutoshi Asai, MD, PhD; \\ Kazunori Kashiwase, MD, PhD; Kazuhisa Kodama, MD, PhD
}

\begin{abstract}
Background: A low ratio of serum eicosapentaenoic acid to arachidonic acid (EPA/AA) has been associated with cardiovascular events. Higher-grade yellow color coronary plaques are associated with higher plaque vulnerability and higher thrombogenic potential. Therefore, the association between EPA/AA ratio and yellow color grade of coronary plaques was examined.
\end{abstract}

\begin{abstract}
Methods and Results: Consecutive patients $(n=54)$ who underwent percutaneous coronary intervention were enrolled in this study. The serum EPA/AA ratio was examined on admission. All patients underwent an angioscopic examination of the culprit vessel to examine the color grade of yellow plaques (0, white; 1 , slight yellow; 2 , yellow; and 3, intense yellow) and the presence of thrombus. Excluding 16 patients with acute coronary syndrome (ACS), 38 patients with stable angina were divided into 2 groups according to their EPA/AA ratio: the low EPA/AA group $(n=19$, EPA/AA ratio $<0.37$ [median]) and the high EPA/AA group $(n=19$, EPA/AA ratio $\geq 0.37)$. The maximum color grade $(2.5 \pm 0.5$ vs. $1.9 \pm 0.9 ; P=0.01)$ of yellow plaques was significantly higher and the number of non-culprit yellow plaques with thrombus $(1.7 \pm 0.8$ vs. $1.2 \pm 1.1 ; \mathrm{P}=0.06)$ tended to be higher in low EPA/AA than in high EPA/AA stable angina patients. Multivariate analysis revealed that the serum EPA level (odds ratio $=0.98,95 \%$ confidence inter$\mathrm{val}=0.96-0.99, \mathrm{P}=0.03$ ) was associated with the presence of grade-3 yellow plaques.
\end{abstract}

Conclusions: A low serum EPA level and a low EPA/AA ratio was associated with high vulnerability of coronary plaques. (Circ J 2011; 75: 2432-2438)

Key Words: Angioscopy; Eicosapentaenoic acid to arachidonic acid ratio; Plaque vulnerability; Thrombus; Yellow plaque

$\mathbf{P}$ revious reports have shown that consumption of fish or fish-oil could protect against cardiovascular events, especially acute coronary syndrome (ACS). Epidemiological and clinical evidence suggests that long-term intake of long-chain n-3 polyunsaturated fatty acids, especially eicosapentaenoic acid (EPA), plays an important role in improving mortality associated with coronary artery disease. ${ }^{1-3}$ A high serum ratio of EPA to arachidonic acid (AA; long-chain $n-6$ polyunsaturated fatty acids) has been associated with a lower incidence of future cardiovascular events and a better outcome. ${ }^{1,2,4-6}$ Furthermore, medication with purified EPA has been demonstrated to reduce future cardiovascular events. ${ }^{7-10}$

A major cause of ACS is the disruption of vulnerable plaques and thrombosis. The vulnerability of coronary plaques is determined mainly by the thickness of the fibrous cap; and the yellow color grade of plaques is inversely associated with the thickness of the fibrous cap and is also directly associated with the frequency of plaques with thrombus. ${ }^{11-13}$ Furthermore, the disrupted yellow plaques are detected at the ACS culprit lesions in $>90 \%$ of cases and thus, yellow plaques are supposed to include both rupture-prone and erosion-prone vulnerable plaques. ${ }^{14-16}$

Therefore, in the present study, we examined the association between the serum EPA/AA ratio and plaque vulnerability evaluated by coronary angioscopy.

\section{Study Patients}

Consecutive patients $(\mathrm{n}=54)$ who underwent percutaneous coronary intervention (PCI) and a coronary angioscopic examination from April 2009 to March 2010 were included in this study. Excluding 16 ACS patients, we divided patients with stable angina into 2 groups according to their serum EPA/AA

Received April 4, 2011; revised manuscript received April 27, 2011; accepted May 20, 2011; released online July 20, 2011 Time for primary review: 6 days

Cardiovascular Division, Osaka Police Hospital, Osaka, Japan

Mailing address: Yasunori Ueda, MD, PhD, FACC, FESC, FJCC, Cardiovascular Division, Osaka Police Hospital, 10-31 Kitayama-cho,

Tennoji-ku, Osaka 543-0035 Japan. E-mail: ueda@oph.gr.jp

ISSN-1346-9843 doi:10.1253/circj.CJ-11-0352

All rights are reserved to the Japanese Circulation Society. For permissions, please e-mail: cj@j-circ.or.jp 


\begin{tabular}{|c|c|c|c|c|c|}
\hline & \multirow[b]{2}{*}{ ACS } & \multicolumn{3}{|c|}{ Stable angina } & \multirow[b]{2}{*}{ P value* } \\
\hline & & All & $\begin{array}{c}\text { Low } \\
\text { EPA/AA }\end{array}$ & $\begin{array}{c}\text { High } \\
\text { EPA/AA }\end{array}$ & \\
\hline Number of patients & 16 & 38 & 19 & 19 & \\
\hline Male gender, n (\%) & $12(75)$ & $30(79)$ & $14(73)$ & $16(84)$ & 0.42 \\
\hline Age, years & $66 \pm 9$ & $69 \pm 8$ & $67 \pm 7$ & $70 \pm 8$ & 0.32 \\
\hline \multicolumn{6}{|l|}{ Coronary risk factors, $\mathbf{n}(\%)$} \\
\hline Current smoking & $12(75)$ & $28(73)$ & $15(79)$ & $13(68)$ & 0.46 \\
\hline Hypertension & $8(50)$ & $27(71)$ & $12(63)$ & $15(79)$ & 0.28 \\
\hline Dislipidemia & $11(68)$ & $28(73)$ & $13(68)$ & $15(79)$ & 0.46 \\
\hline Diabetes mellitus & $3(18)$ & $18(47)$ & $11(58)$ & $7(36)$ & 0.19 \\
\hline Prior history of ACS, $n$ (\%) & $2(13)$ & $18(47)$ & $9(47)$ & $9(47)$ & 1.0 \\
\hline \multicolumn{6}{|l|}{ Medications, n (\%) } \\
\hline Aspirin & $2(12)$ & $28(73)$ & $13(68)$ & $15(79)$ & 0.46 \\
\hline Ticlopidine/Clopidogrel & $1(6)$ & $17(45)$ & $8(42)$ & $9(47)$ & 0.74 \\
\hline Statin & $3(18)$ & $24(63)$ & $15(79)$ & $13(68)$ & 0.46 \\
\hline ACEI/ARB & $1(6)$ & $20(52)$ & $9(47)$ & $11(58)$ & 0.51 \\
\hline$\beta$-blocker & $1(6)$ & $18(47)$ & $8(42)$ & $10(52)$ & 0.51 \\
\hline \multicolumn{6}{|l|}{ Serum lipid profile, mg/dl } \\
\hline Total cholesterol & $211 \pm 39$ & $176 \pm 24$ & $171 \pm 19$ & $182 \pm 27$ & 0.15 \\
\hline LDL cholesterol & $133 \pm 30$ & $99 \pm 21$ & $102 \pm 21$ & $95 \pm 22$ & 0.32 \\
\hline HDL cholesterol & $45 \pm 10$ & $45 \pm 9$ & $44 \pm 8$ & $46 \pm 9$ & 0.43 \\
\hline Triglyceride & $166 \pm 90$ & $185 \pm 127$ & $148 \pm 62$ & $221 \pm 62$ & 0.07 \\
\hline LDL/HDL ratio & $3.03 \pm 0.86$ & $2.24 \pm 0.58$ & $2.36 \pm 0.56$ & $2.11 \pm 0.59$ & 0.19 \\
\hline \multicolumn{6}{|l|}{ Polyunsaturated fatty acid, $\mu \mathrm{g} / \mathrm{ml}$} \\
\hline EPA & $63.0 \pm 41$ & $68.1 \pm 40$ & $36.3 \pm 15$ & $99.9 \pm 30$ & $<0.001$ \\
\hline$A A$ & $147 \pm 307$ & $165 \pm 30$ & $170 \pm 26$ & $160 \pm 34$ & 0.31 \\
\hline $\mathrm{DHA}$ & $186 \pm 30$ & $139 \pm 50$ & $112 \pm 34$ & $166 \pm 50$ & $<0.001$ \\
\hline DHLA & $33 \pm 10$ & $37 \pm 10$ & $41 \pm 10$ & $32 \pm 9$ & 0.002 \\
\hline EPA/AA ratio & $0.46 \pm 0.34$ & $0.42 \pm 0.26$ & $0.21 \pm 0.08$ & $0.63 \pm 0.20$ & - \\
\hline C-reactive protein, $\mathrm{mg} / \mathrm{dl}$ & $0.21 \pm 0.23$ & $0.19 \pm 0.31$ & $0.22 \pm 0.37$ & $0.17 \pm 0.24$ & 0.58 \\
\hline Culprit vessel, n (\%) & & & & & 0.35 \\
\hline LAD & $11(69)$ & $12(32)$ & $4(21)$ & $8(42)$ & \\
\hline LCX & $0(0)$ & $3(8)$ & $2(10)$ & $1(5)$ & \\
\hline RCA & $5(31)$ & $23(60)$ & $13(69)$ & $10(53)$ & \\
\hline \multicolumn{6}{|l|}{ Culprit lesion } \\
\hline Diameter stenosis (\%) & $97 \pm 4$ & $88 \pm 15$ & $88 \pm 15$ & $87 \pm 16$ & 0.82 \\
\hline AHA/ACC classification type B2/C, n (\%) & $11(69)$ & $25(66)$ & $13(68)$ & $12(63)$ & 0.73 \\
\hline Diagnosis, n (\%) & & & & & - \\
\hline Stable angina & $0(0)$ & $38(100)$ & $19(100)$ & $19(100)$ & \\
\hline Unstable angina & $5(31)$ & $0(0)$ & $0(0)$ & $0(0)$ & \\
\hline NSTEMI & $4(25)$ & $0(0)$ & $0(0)$ & $0(0)$ & \\
\hline STEMI & $7(44)$ & $0(0)$ & $0(0)$ & $0(0)$ & \\
\hline
\end{tabular}

${ }^{*}$ Patients with low vs. high EPA/AA ratio among stable angina patients.

ACS, acute coronary syndrome; EPA/AA, eicosapentaenoic acid (EPA) to arachidonic acid (AA); ACEI, angiotensinconverting enzyme inhibitor; ARB, angiotensin receptor blocker; LDL, low-density lipoprotein; HDL, high-density lipoprotein; DHA, docosahexaenoic acid; DHLA, dihomo- $\gamma$-linolenic acid; LAD, left anterior descending coronary artery; LCX, left circumflex coronary artery; RCA, right coronary artery; NSTEMI, non-ST segment elevation myocardial infarction; STEMI, ST segment elevation myocardial infarction.

ratio: patients with a low EPA/AA ratio ( $\mathrm{n}=19, \mathrm{EPA} / \mathrm{AA}$ ratio $<0.37$ [median]) and patients with a high EPA/AA ratio $(n=19$, EPA/AA ratio $\geq 0.37$ [median]). Angioscopic findings were compared between these 2 groups. The data of ACS patients were also presented as a reference.

Blood samples were acquired from all patients in a fasting state on admission to measure polyunsaturated fatty acids, that is, EPA, AA, docosahexaenoic acid (DHA), and dihomogamma-linolenic acid (DHLA). The measurement was performed by gas chromatography at the central laboratory (SRL,
Tokyo, Japan) using a gas chromatograph (GC-2010; Shimadzu, Kyoto, Japan) and a capillary column (BPX90; Wako, Osaka, Japan).

ACS includes acute MI defined by The Joint European Society of Cardiology/American College of Cardiology Committee and unstable angina, defined according to the Braunwald classification. Hypertensive patients were defined as those with a systemic arterial pressure of $>140 / 90 \mathrm{mmHg}$ or those already taking anti-hypertensive drugs. Diabetic patients were defined as those with a fasting blood glucose of $>126 \mathrm{mg} / \mathrm{dl}$ or those 

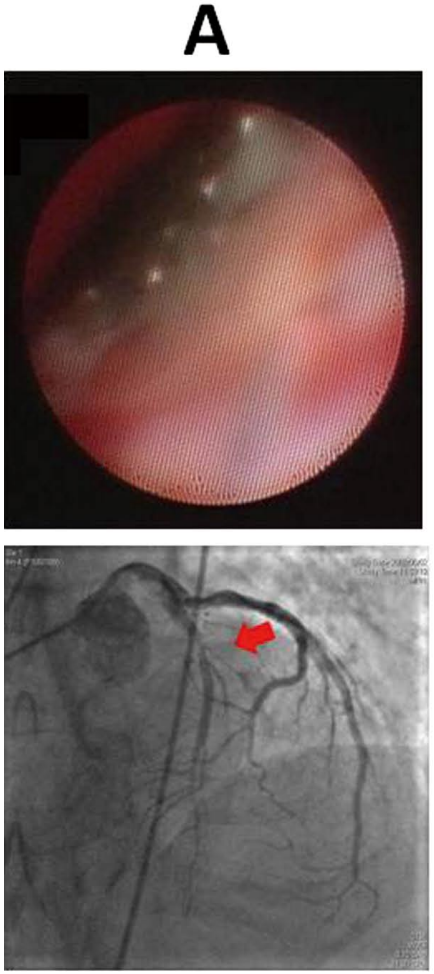

Anterior AMI $E P A / A A=0.30$
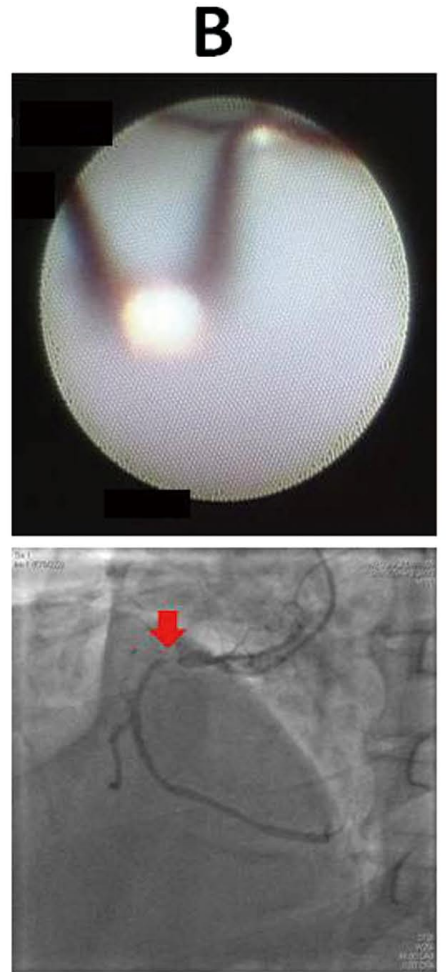

Stable Angina $E P A / A A=0.82$
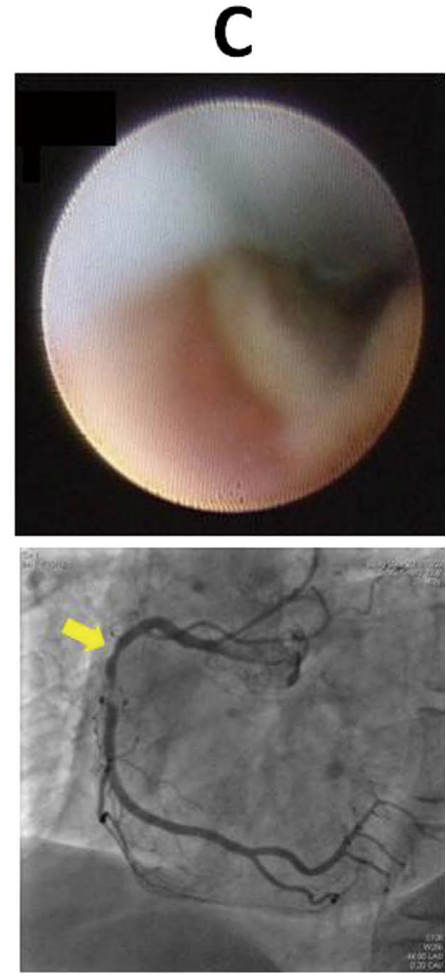

Stable Angina $E P A / A A=0.23$

Figure 1. A representative case from the acute coronary syndrome patient group, the low eicosapentaenoic acid to arachidonic acid (EPA/AA) stable angina patient group, and the high EPA/AA stable angina patient group. (A) An angioscopic image of a stented culprit lesion (red arrow) in the patients with anterior acute myocardial infarction (AMI). Grade-3 yellow plaque with red thrombus was observed at the culprit lesion in the proximal left anterior descending coronary artery. The maximum yellow color grade was 3 and the number of non-culprit yellow plaques with thrombus was 6 . The EPA/AA ratio was 0.30. (B) An angiosopic image of a stented culprit lesion (red arrow) in the stable angina patients with a high EPA/AA ratio. A white (grade-0) vessel wall was observed and no yellow plaque was detected in the vessel. The maximum yellow color grade was 0 and the number of nonculprit yellow plaques with thrombus was 0. The EPA/AA ratio was 0.82. (C) An angioscopic image of yellow plaque in the nonculprit segments (yellow arrow) in the stable angina patients with a low EPA/AA ratio. Grade-3 yellow plaque with thrombus was observed in the non-culprit segment. The maximum yellow color grade was 3 and the number of non-culprit yellow plaques with thrombus was 1. The EPA/AA ratio was 0.23 .

already taking oral drugs for diabetes mellitus or receiving insulin therapy. Patients with dyslipidemia were defined as those with a LDL cholesterol $>140 \mathrm{mg} / \mathrm{dl}$, HDL cholesterol $<40 \mathrm{mg} / \mathrm{dl}$, triglyceride $>150 \mathrm{mg} / \mathrm{dl}$ or those already taking lipidlowering drugs. No patients in the present study were taking the purified EPA drug, ethyl eicosapentate. Written informed consent was acquired from all patients. This protocol was approved by the Osaka Police Hospital Ethical Committee.

\section{Coronary Intervention and Angioscopic Examination}

Catheterization was performed by a femoral, brachial, or radial artery approach using a 6- or 7-Fr sheath and catheters. Intravenous heparin $(100 \mathrm{U} / \mathrm{kg})$ was administered at the beginning of catheterization, and an additional dose was repeated at the time of PCI as a routine protocol in our hospital. An angioscopic examination was performed after stent implantation at the culprit lesion. Oral aspirin ( $100 \mathrm{mg} /$ day) and ticlopidine $(75 \mathrm{mg} /$ day $)$ were started as soon as possible in the stable angina patients. A loading dose of oral aspirin $(300 \mathrm{mg})$ and ticlopidine $(300 \mathrm{mg}$ ) were administered before catheterization in the ACS patients.

An angioscopic examination of the whole culprit vessel was performed to evaluate yellow plaques. The angioscope RX3310A and MV-5010A (Machida, Tokyo, Japan) and optic fiber DAG-2218LN (Machida) were used. Angioscopic observations were made while blood was cleared away from view by the injection of $3 \%$ dextran-40, as previously reported. ${ }^{17}$ The color grade of detected yellow plaques was determined according to the standard colors ( 0 , white; 1 , slight yellow; 2 , yellow; and 3, intensive yellow), as previously reported. ${ }^{14}$ The number of detected yellow plaques and maximum color grade of those plaques were determined for each patient. The presence or absence of thrombus adhering to the plaques was also determined, and the number of non-culprit yellow plaques with thrombus was counted for each patient. The presence or absence of thrombus at the culprit yellow plaques was excluded from analysis as it might be influenced by the PCI procedures. Thrombus was defined as white or red material that had a cotton-like or ragged appearance or that presented fragmentation 

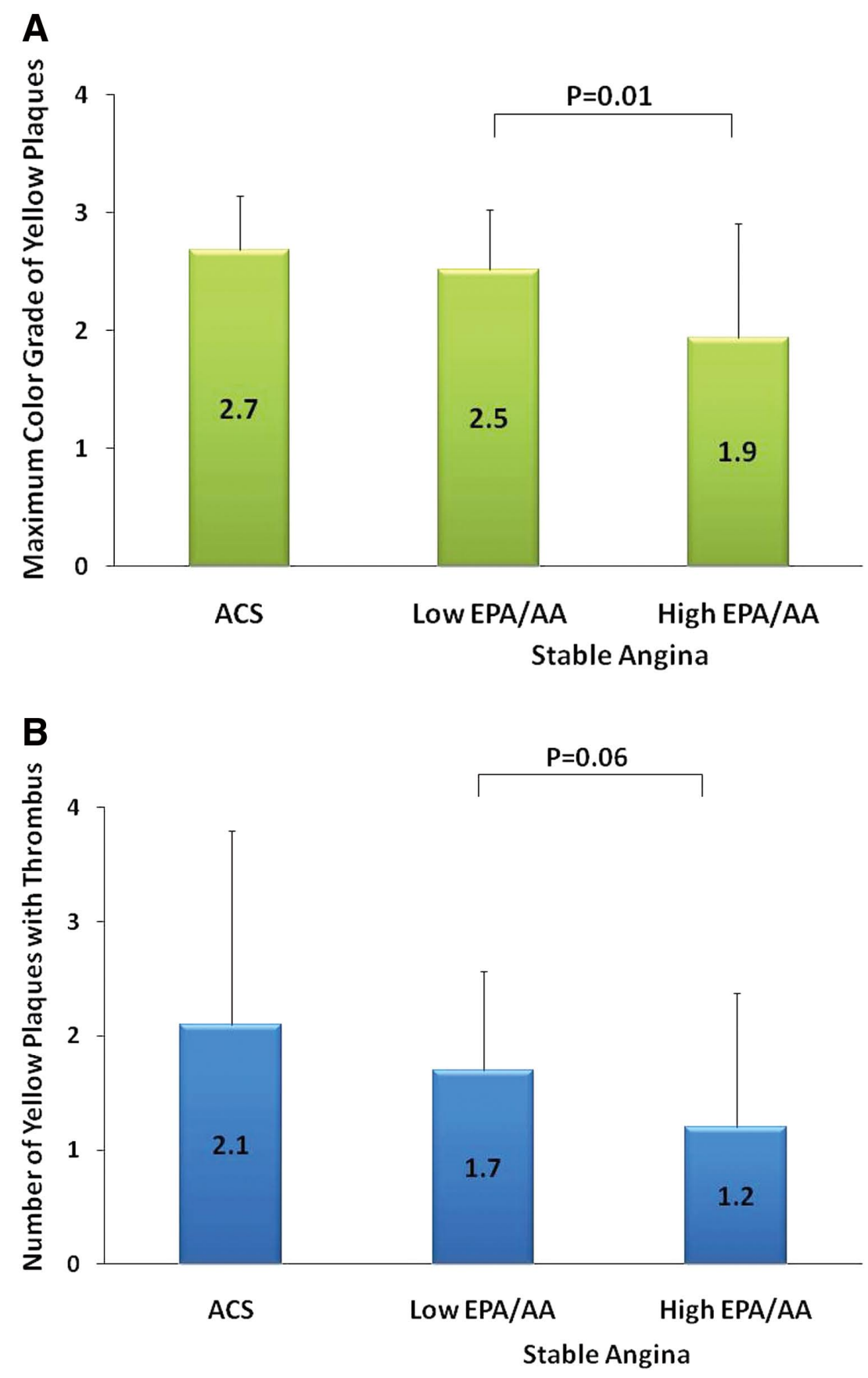

Figure 2. Maximum color grade and number of non-culprit yellow plaques with thrombus. The maximum color grade $(\mathbf{A})$ was higher in the low than in the high eicosapentaenoic acid to arachidonic acid (EPA/AA) stable angina patients. The number of non-culprit yellow plaques with thrombus (B) tended to be higher in the low than in the high EPA/AA stable angina patients. ACS, acute coronary syndrome.

with or without protrusion into the lumen or was adherent to the luminal surface. Angioscopic images were evaluated by 2 experienced observers who were unaware of the patients' background. In the case of disagreement, a joint reading was performed and a consensus decision was reached. The interand intra-observer reproducibility for the interpretation of angioscopic images was $85 \%$ and $95 \%$ for plaque color, and $90 \%$ and $100 \%$ for thrombus, respectively.

\section{Statistical Analysis}

Continuous data were presented as mean \pm SD. Comparisons between low and high EPA/AA groups were done by using an unpaired Student's t-test or a chi-square test. Multivariate step- wise logistic regression analysis was performed to analyze the factors associated with the presence of grade-3 yellow plaque (maximum color grade =3) among EPA, AA, DHA, LDL cholesterol, HDL cholesterol, and C-reactive protein. The ACS group was presented only as a reference and was not included in any statistical analysis. A P value $<0.05$ was regarded as statistically significant. Analysis was done with Dr SPSS II for Windows (SPSS Japan Inc, Tokyo, Japan).

\section{Results}

Patients' characteristics are presented in Table. Among stable angina patients, the EPA and EPA/AA ratio and also the DHA 


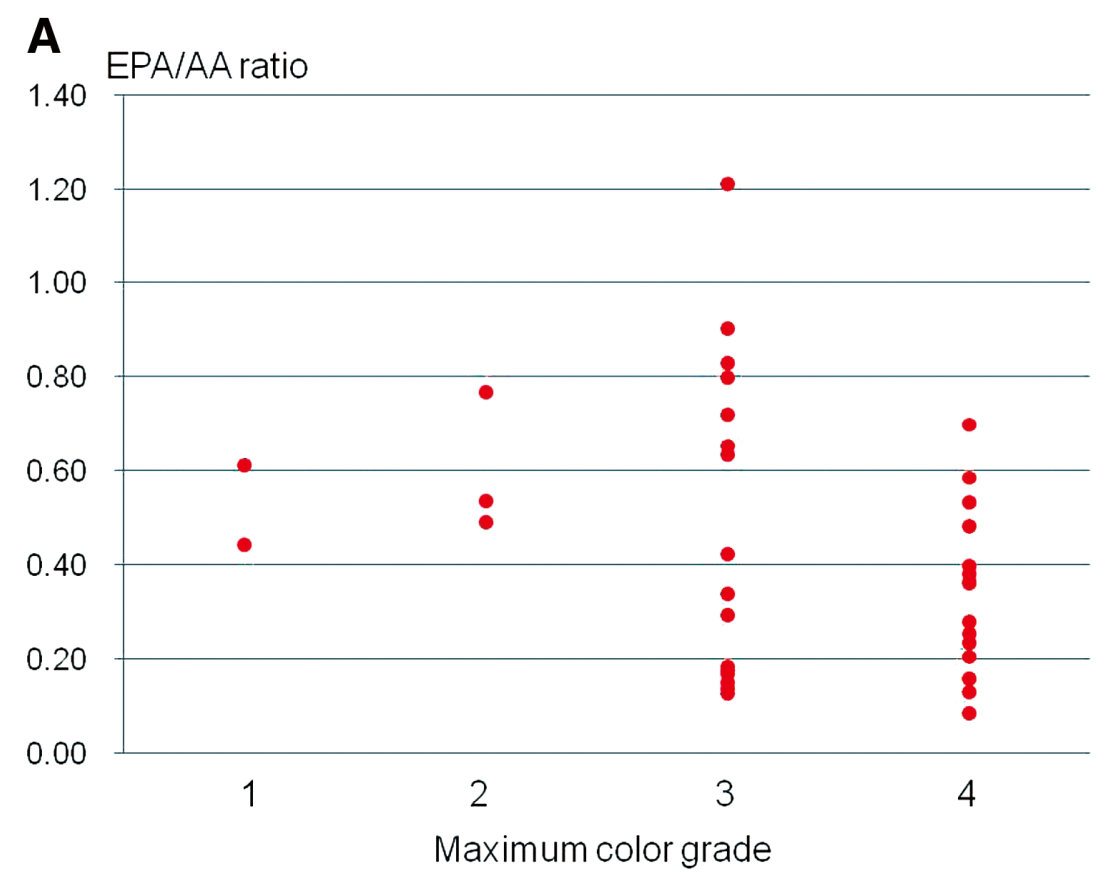

B

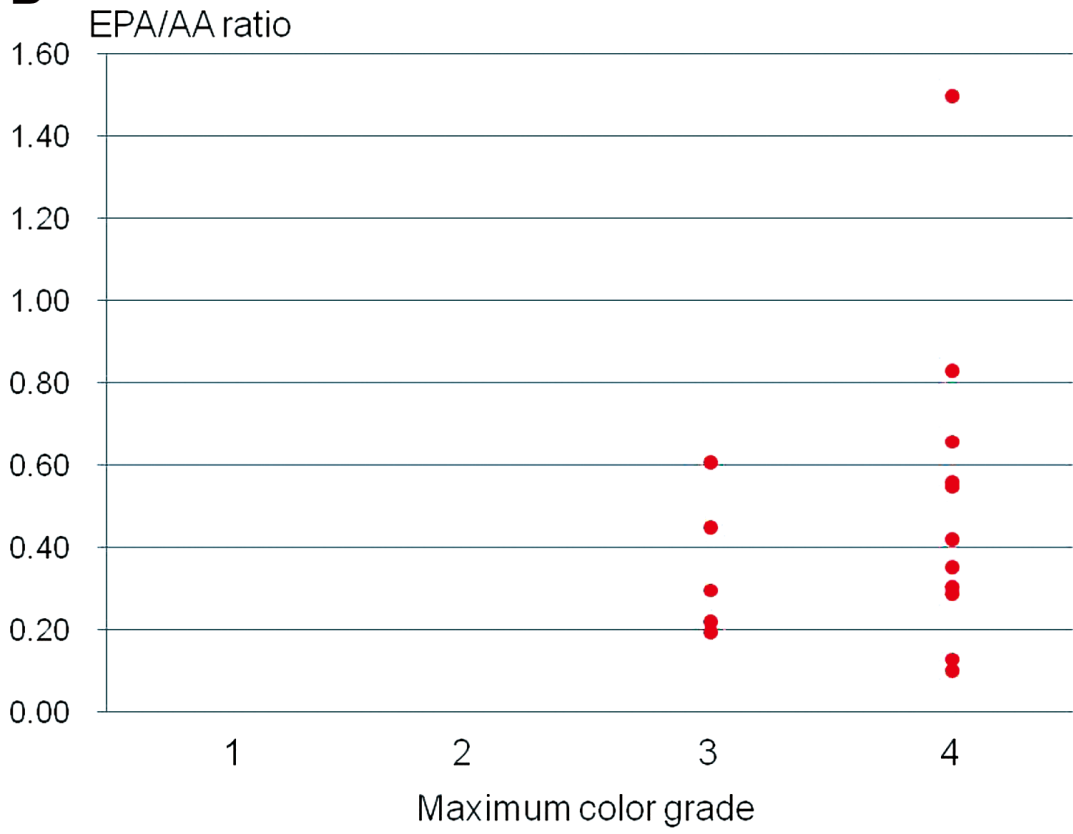

Figure 3. Scatter plot to show the correlation between maximum color grade and eicosapentaenoic acid to arachidonic acid (EPA/AA) ratio. Scatter plot for stable angina patients (A) and acute coronary syndrome (ACS) patients $(\mathrm{B})$ are presented. The maximum color grade had weak but a significant correlation with the EPA/AA ratio $(R=-0.29, P=0.03)$ in stable angina patients. In ACS patients, all patients had rather high maximum color grades of 2 or 3 , and there appeared to be no correlation between maximum color grade and EPA/AA ratio.

level was significantly lower in the low than in the high EPA/ AA group. No other background factors were different between the low and high EPA/AA groups. Representative cases from 3 groups of patients (ACS patients, low EPA/AA stable angina patients, and high EPA/AA stable angina patients) are presented in Figure 1.

The number of detected yellow plaques was not different between the low and the high EPA/AA ratio stable angina patients $(3.1 \pm 1.6$ vs. $3.0 \pm 2.3, \mathrm{P}=0.4)$, while it was $4.5 \pm 2.6$ in ACS patients. The maximum color grade ( $2.5 \pm 0.5$ vs. $1.9 \pm 0.9$; $\mathrm{P}=0.01$ ) of yellow plaques was significantly higher in the low than in the high EPA/AA stable angina patients (Figure 2A), while it was $2.7 \pm 0.5$ in ACS patients. The number of plaques with thrombus $(1.7 \pm 0.8$ vs. $1.2 \pm 1.1 ; \mathrm{P}=0.06)$ in the non-culprit segments tended to be larger in the low than in the high EPA/ AA stable angina patients (Figure 2B), while it was 2.1 1.7 in ACS patients.

The maximum color grade had a weak but significant correlation with EPA $(\mathrm{R}=-0.40, \mathrm{P}=0.006)$, EPA/AA ratio $(\mathrm{R}=$ $-0.29, \mathrm{P}=0.03)$, and DHA $(\mathrm{R}=-0.40, \mathrm{P}=0.007)$. The correlation between maximum color grade and EPA/AA ratio is presented as scatter plot in Figure 3. The number of plaques with thrombus in the non-culprit segments also had a weak but significant correlation with EPA $(\mathrm{R}=-0.45, \mathrm{P}=0.002), \mathrm{EPA} /$ AA ratio $(\mathrm{R}=-0.37, \mathrm{P}=0.01)$, and $\mathrm{DHA}(\mathrm{R}=-0.27, \mathrm{P}=0.04)$.

Multivariate analysis revealed that the serum EPA level 
(odds ratio $=0.98,95 \%$ confidence interval $=0.96-0.99, \mathrm{P}=0.03$ ) was significantly associated with the presence of grade- 3 yellow plaque (maximum color grade =3), when EPA, AA, DHA, LDL cholesterol, HDL cholesterol, and C-reactive protein were included in the analysis.

\section{Discussion}

In the present study, we evaluated coronary plaque vulnerability by the maximum color grade of yellow plaques and the number of already thrombogenic/disrupted yellow plaques in the non-culprit segments, and revealed its association with the EPA/AA ratio among stable angina patients. Additionally, the plaque vulnerability in low EPA/AA stable angina patients appeared similar to that in ACS patients.

The additive effect of EPA therapy and antiplatelet therapy has been reported. ${ }^{18} \mathrm{EPA}$ therapy reduces serum small dense LDL and high sensitive C-reactive protein, ${ }^{19,20}$ and the antiinflammatory effect of EPA is considered to be an important mechanism to protect against atherosclerotic disease. ${ }^{21} \mathrm{~A}$ majority of patients with stable angina were taking aspirin and statin and had a relatively low LDL cholesterol level in the present study; however, those patients with a low EPA/AA ratio had higher plaque vulnerability than those with a high EPA/AA ratio. Therefore, EPA therapy might contribute to the stabilization of coronary plaques and prevention of cardiovascular events through different mechanisms from statins or antiplatelet therapy.

Although the extent of atherosclerosis has been associated with the serum EPA level, as found by a study with coronary computed tomography, ${ }^{22}$ the present study would be the first to have examined the association between EPA/AA ratio and the level of plaque vulnerability. Judging from the result of multivariate analysis in which markers of serum fatty acid (EPA, AA, and DHA), cholesterol (LDL and HDL cholesterol), and inflammation (C-reactive protein) were included, a low serum EPA level was significantly associated with the presence of grade- 3 yellow plaques, which are regarded as vulnerable plaques and compatible with thin-cap fibroatheroma. ${ }^{13,15}$ Although the correlation between maximum color grade and the EPA/AA ratio was weak, this weak correlation, judging from the scatter plot in Figure 3, might be partly due to the lack of rather healthy patients who had low maximum color grade and a high EPA/ AA ratio. Because maximum color grade is determined by multiple factors, it is not surprising that the correlation with each of them is weak. In the present study, LDL cholesterol was not significantly associated with the color grade by multivariate analysis. This might be because the level of serum LDL cholesterol was well controlled by statin treatment in the present study patients, as shown in Table. We have previously demonstrated that statin treatment reduces the color grade of coronary plaques and that the reduction of color grade is not associated with the achieved LDL cholesterol level. ${ }^{23}$ In ACS patients, all patients had rather high maximum color grades of 2 or 3 , and there appeared to be no correlation between maximum color grade and EPA/AA ratio. Culprit and non-culprit yellow plaques in ACS patients are known to reduce their yellow color grade during follow up after the onset of ACS. ${ }^{14,24}$ Therefore, a low serum EPA level and a low EPA/AA ratio would be associated with high coronary plaque vulnerability; and EPA therapy might reduce cardiovascular events by stabilizing coronary plaques.

\section{Study Limitations}

The number of patients was too small in the present study to analyze adequately the contributing factors for the maximum color grade. In particular, judging from the scatter plot in Figure 3, healthy stable angina patients with a low maximum color grade and a high EPA/AA ratio would be required in order to strengthen our conclusion. This group of rather healthy patients who did not require PCI was not included in the present study because we included only the patients who received PCI as a study design. Therefore, our findings should be confirmed by further investigations. All 3 coronary vessels were not examined. An angioscopic examination was performed after stent implantation so that the whole vessel could be observed; however, we excluded the evaluation of thrombus at the stented lesion to minimize the influence of a PCI procedure on the angioscopic findings.

\section{Conclusion}

Low serum EPA level and a low EPA/AA ratio were associated with the high vulnerability of coronary plaques.

\section{Disclosures}

None.

\section{References}

1. Dyerberg J, Bang HO, Stoffersen E, Moncada S, Vane JR. Eicosapentaenoic acid and prevention of thrombosis and atherosclerosis? Lancet 1978; 2: 117-119.

2. Nakamura T, Azuma A, Kuribayashi T, Sugihara H, Okuda S, Nakagawa M. Serum fatty acid levels, dietary style and coronary heart disease in three neighbouring areas in Japan: The Kumihama study. Br J Nutr 2003; 89: 267-272.

3. Marik PE, Varon J. Omega-3 dietary supplements and the risk of cardiovascular events: A systematic review. Clin Cardiol 2009; 32: $365-372$.

4. Domei T, Ueeda M, Takaya Y, Ohnishi N, Takaishi A, Imai M, et al. The balance of $n 6 / n 3$ polyunsaturated fatty acid (PUFAs) is important factor of prognosis after acute myocardial infarction. Circulation 2006; 114: II-463.

5. Domei T, Kuramitsu S, Ando K, Soga Y, Arita T, Shirai S, et al. The relationship between the ratio of serum eicosapentaenoic acid (EPA) to arachidonic acid (AA) with major cardiac events in patients undergoing elective percutaneous coronary intervention. Circulation 2009; 120: $\mathrm{S} 426$.

6. Matsuzaki M, Yokoyama M, Saito Y, Origasa H, Ishikawa Y, Oikawa $\mathrm{S}$, et al; for the JELIS Investigators, Japan. Incremental effects of eicosapentaenoic acid on cardiovascular events in statin-treated patients with coronary artery disease: Secondary prevention analysis from JELIS. Circ J 2009; 73: 1283-1290.

7. Lavie CJ, Milani RV, Mehra MR, Ventura HO. Omega-3 polyunsaturated fatty acids and cardiovascular diseases. J Am Coll Cardiol 2009; 54: 585-594.

8. Saito Y, Yokoyama M, Origasa H, Matsuzaki M, Matsuzawa Y, Ishikawa Y, et al; JELIS Investigators, Japan. Effects of EPA on coronary artery disease in hypercholesterolemic patients with multiple risk factors: Sub-analysis of primary prevention cases from the Japan EPA Lipid Intervention Study (JELIS). Atherosclerosis 2008; 200: $135-140$

9. Mita T, Watada H, Ogihara T, Nomiyama T, Ogawa O, Kinoshita J, et al. Eicosapentaenoic acid reduces the progression of carotid intimamedia thickness in patients with type 2 diabetes. Atherosclerosis 2007 ; 191: $162-167$.

10. Tanaka K, Ishikawa Y, Yokoyama M, Origasa H, Matsuzaki M, Saito $\mathrm{Y}$, et al; JELIS Investigators, Japan. Reduction in the recurrence of stroke by eicosapentaenoic acid for hypercholesterolemic patients: Subanalysis of the JELIS trial. Stroke 2008; 39: 2052-2058.

11. Ueda Y, Ohtani T, Shimizu M, Hirayama A, Kodama K. Assessment of plaque vulnerability by angioscopic classification of plaque color. Am Heart J 2004; 148: 333-335.

12. Virmani R, Burke AP, Kolodgie FD, Farb A. Vulnerable plaque: The pathology of unstable coronary lesions. J Interv Cardiol 2002; 15: $439-446$.

13. Kubo T, Imanishi T, Takarada S, Kuroi A, Ueno S, Yamano T, et al. Implication of plaque color classification for assessing plaque vulnerability: A coronary angioscopy and optical coherence tomography investigation. JACC Cardiovasc Interv 2008; 1: 74-80. 
14. Ueda Y, Asakura M, Yamaguchi O, Hirayama A, Hori M, Kodama $\mathrm{K}$. The healing process of infarct-related plaques: Insights from 18 months of serial angioscopic follow-up. J Am Coll Cardiol 2001; 38: $1916-1922$.

15. Ueda Y, Ogasawara N, Matsuo K, Hirotani S, Kashiwase K, Hirata A, et al. Acute coronary syndrome: Insight from angioscopy. Circ $J$ 2010; 74: 411-417.

16. Sanidas EA, Maehara A, Mintz GS, Kashiyama T, Guo J, Pu J, et al. Angioscopic and virtual histology intravascular ultrasound characteristics of culprit lesion morphology underlying coronary artery thrombosis. Am J Cardiol 2011; 107: 1285-1290.

17. Ueda Y, Nanto S, Komamura K, Kodama K. Neointimal coverage of stents in human coronary arteries observed by angioscopy. $J \mathrm{Am}$ Coll Cardiol 1994; 23: 341 - 346.

18. Gajos G, Rostoff P, Undas A, Piwowarska W. Effects of polyunsaturated omega-3 fatty acids on responsiveness to dual antiplatelet therapy in patients undergoing percutaneous coronary intervention: The OMEGA-PCI (OMEGA-3 fatty acids after PCI to modify responsiveness to dual antiplatelet therapy) study. J Am Coll Cardiol 2010; 55: $1671-1678$

19. Satoh N, Shimatsu A, Kotani K, Sakane N, Yamada K, Suganami T, et al. Purified eicosapentaenoic acid reduces small dense LDL, rem- nant lipoprotein particles, and C-reactive protein in metabolic syndrome. Diabetes Care 2007; 30: 144-146.

20. Yokoyama M, Origasa H, Matsuzaki M, Matsuzawa Y, Saito Y, Ishikawa Y, et al; Japan EPA lipid intervention study (JELIS) Investigators. Effects of eicosapentaenoic acid on major coronary events in hypercholesterolaemic patients (JELIS): A randomised open-label, blinded endpoint analysis. Lancet 2007; 369: 1090-1098.

21. Calder PC. $\mathrm{N}-3$ polyunsaturated fatty acids and inflammation: From molecular biology to the clinic. Lipids 2003; 38: 343-352.

22. Ueeda M, Doumei T, Takaya Y, Shinohata R, Katayama Y, Ohnishi $\mathrm{N}$, et al. Serum N-3 polyunsaturated fatty acid levels correlate with the extent of coronary plaques and calcifications in patients with acute myocardial infarction. Circ J 2008; 72: 1836-1843.

23. Kodama K, Komatsu S, Ueda Y, Takayama T, Yajima J, Nanto S, et al. Stabilization and regression of coronary plaques treated with pitavastatin proven by angioscopy and intravascular ultrasound: The TOGETHAR trial. Circ J 2010; 74: 1922-1928.

24. Okada K, Ueda Y, Matsuo K, Nishio M, Hirata A, Kashiwase K, et al. Frequency and healing of nonculprit coronary artery plaque disruptions in patients with acute myocardial infarction. Am J Cardiol 2011; 107: 1426-1429. 